\title{
Electrical properties of extended defects in strain relaxed GeSn
}

Somya Gupta, Eddy Simoen, Roger Loo, Yosuke Shimura, Clement Porret, Federica Gencarelli, Kristof Paredis, Hugo Bender, Johan Lauwaert, Henk Vrielinck, and Marc Heyns

Citation: Appl. Phys. Lett. 113, 022102 (2018); doi: 10.1063/1.5034573

View online: https://doi.org/10.1063/1.5034573

View Table of Contents: http://aip.scitation.org/toc/apl/113/2

Published by the American Institute of Physics

\section{Articles you may be interested in}

Control of dipole properties in high-k and $\mathrm{SiO}_{2}$ stacks on $\mathrm{Si}$ substrates with tricolor superstructure Applied Physics Letters 113, 012103 (2018); 10.1063/1.5034494

Artificial electronic synapse characteristics of a Ta/Ta2 $\mathrm{O}_{5-x} / \mathrm{Al}_{2} \mathrm{O}_{3} / \mathrm{lnGaZnO} 4$ memristor device on flexible stainless steel substrate

Applied Physics Letters 113, 013503 (2018); 10.1063/1.5027776

An approach to the measurement of the nonlinear refractive index of very short lengths of optical fibers Applied Physics Letters 113, 011108 (2018); 10.1063/1.5035146

Evidence for a surface anomaly during the cubic-tetragonal phase transition in $\mathrm{BaTiO}_{3}(001)$

Applied Physics Letters 113, 022901 (2018); 10.1063/1.5030498

Contributions of the lead-bromine weighted bands to the occupied density of states of the hybrid tri-bromide perovskites

Applied Physics Letters 113, 022101 (2018); 10.1063/1.5024527

Graphdiyne under pressure: A Raman study

Applied Physics Letters 113, 021901 (2018); 10.1063/1.5023619

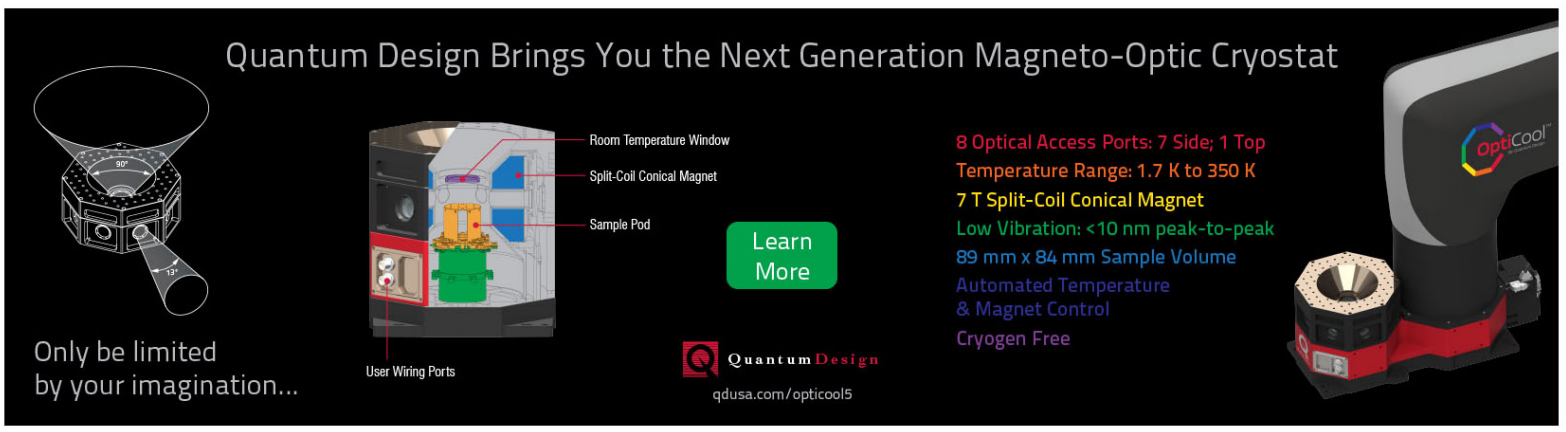




\title{
Electrical properties of extended defects in strain relaxed GeSn
}

\author{
Somya Gupta, ${ }^{1,2, a)}$ Eddy Simoen, ${ }^{1,3}$ Roger Loo, ${ }^{1}$ Yosuke Shimura, ${ }^{1,4}$ Clement Porret, ${ }^{1}$ \\ Federica Gencarelli, ${ }^{1}$ Kristof Paredis, ${ }^{1}$ Hugo Bender, ${ }^{1}$ Johan Lauwaert, ${ }^{5}$ Henk Vrielinck, ${ }^{3}$ \\ and Marc Heyns ${ }^{1,2}$ \\ ${ }^{1}$ Imec, Kapeldreef 75 , Leuven B-3001, Belgium \\ ${ }^{2}$ KU Leuven, Leuven B-3001, Belgium \\ ${ }^{3}$ Ghent University, Ghent B-9000, Belgium \\ ${ }^{4}$ Shizuoka University, Hamamatsu 432-8011, Japan \\ ${ }^{5}$ Ghent University, Zwijnaarde B-9052, Belgium
}

(Received 12 April 2018; accepted 21 June 2018; published online 9 July 2018)

\begin{abstract}
We report the electrical properties of $60^{\circ}$ dislocations originating from the $+1.2 \%$ lattice mismatch between an unintentionally doped, $315 \mathrm{~nm}$ thick $\mathrm{Ge}_{0.922} \mathrm{Sn}_{0.078}$ layer $(58 \%$ relaxed) and the underlying Ge substrate, using deep level transient spectroscopy. The $60^{\circ}$ dislocations are found to be split into Shockley partials, binding a stacking fault. The dislocations exhibit a band-like distribution of electronic states in the bandgap, with the highest occupied defect state at $\sim \mathrm{E}_{\mathrm{V}}+0.15 \mathrm{eV}$, indicating no interaction with point defects in the dislocation's strain field. A small capture crosssection of $1.5 \times 10^{-19} \mathrm{~cm}^{2}$ with a capture barrier of $60 \mathrm{meV}$ is observed, indicating a donor-like nature of the defect-states. Thus, these dislocation-states are not the source of unintentional p-type doping in the $\mathrm{Ge}_{0.922} \mathrm{Sn}_{0.078}$ layer. Importantly, we show that the resolved $60^{\circ}$ dislocation-states act as a source of leakage current by thermally generating minority electrons via the Shockley-ReadHall mechanism. Published by AIP Publishing. https://doi.org/10.1063/1.5034573
\end{abstract}

$\mathrm{Ge}_{1-\mathrm{x}} \mathrm{Sn}_{\mathrm{x}}$, an alloy of Ge and $\alpha-\mathrm{Sn}$, has instigated a transcendental interest in below $10 \mathrm{~nm}$ technology nodes due to two notable characteristics. ${ }^{1-3}$ Firstly, $\mathrm{Ge}_{1-x} \mathrm{Sn}_{\mathrm{x}}$ has a larger bulk lattice constant in comparison to $\mathrm{Ge}$ and $\mathrm{Si}$. As a result, $\mathrm{Ge}_{1-\mathrm{x}} \mathrm{Sn}_{\mathrm{x}}$ strain relaxed buffers ${ }^{3}$ could allow the tuning of longitudinal tensile strain in Ge channels for n-type fin field effect transistors (FinFETs). ${ }^{3,4}$ Secondly, the band structure of $\mathrm{Ge}_{1-\mathrm{x}} \mathrm{Sn}_{\mathrm{x}}$ can be tuned as a function of the misfit strain and the Sn content, and a transition from the indirect-L to direct- $\Gamma$ bandgap can occur. ${ }^{5,6}$ The possibility of a narrow direct bandgap makes it alluring for p-type tunnel FETs (TFETs) ${ }^{7}$ and infrared optical components like photo-detectors, ${ }^{8}$ light emitting diodes and lasers. ${ }^{2,5}$ However, the Sn content required to observe indirect to direct transition increases with the magnitude of compressive strain, ${ }^{2,5,6}$ thereby impelling the use of strain-relaxed $\mathrm{Ge}_{1-x} \mathrm{Sn}_{\mathrm{x}}$. The high compressive strain due to the lattice mismatch between $\mathrm{Ge}_{1-\mathrm{x}} \mathrm{Sn}_{\mathrm{x}}$ and Ge/ virtual-Ge or $\mathrm{Si}$ substrates is released above a critical epitaxial layer thickness. The relaxation usually occurs by introduction of extended defects (EDs), comprising linear defects (dislocations), planar defects (e.g., stacking faults, twins) and 3-dimensional islands. ${ }^{2,9}$ Though strain-relaxed $\mathrm{Ge}_{1-\mathrm{x}} \mathrm{Sn}_{\mathrm{x}}$ is advantageous for certain device applications, the EDs are detrimental to both optical and electrical devices. EDs are known to (i) generate excess current in transport based devices via Shockley-Read-Hall (SRH) generation, trap assisted tunneling and 1-D conduction, ${ }^{10}$ (ii) act as powerful trap/recombination sites, ${ }^{11,12}$ thereby degrading the efficiency of lasers and photodetectors, and (iii) alter free carrier concentrations. ${ }^{12,13}$ For quantifying the impact of ED on devices' performances, their electronic signature is often obtained using deep level transient spectroscopy (DLTS). ${ }^{14-18}$ The defect's electronic

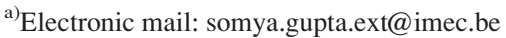

signature consists of, but is not limited to, the activation energy $\left(E_{T}\right)$, the capture cross-section $\left(\sigma_{n}, \sigma_{p}\right)$, the defect concentration $\left(N_{T}\right)$, the donor-acceptor nature, and the field dependent emission.

Rather surprisingly, very little work has been done in obtaining the signature of defects in $\mathrm{Ge}_{1-\mathrm{x}} \mathrm{Sn}_{\mathrm{x}}$ epitaxial layers. Ryu et al. ${ }^{19}$ have studied strain-relaxed $\mathrm{Ge}_{0.94} \mathrm{Sn}_{0.06}$ grown on a Si substrate and suggested the presence of 2 shallow acceptor-like defect-states with activation energies of $7.5 \mathrm{meV}$ and $140 \mathrm{meV}$ based on the experimental fitting of the Hall mobility. Takeuchi et al..$^{20}$ have studied strainrelaxed $\mathrm{Ge}_{1-x} \mathrm{Sn}_{\mathrm{x}}$ for $0.013 \leq x \leq 0.032$ grown on $\mathrm{Si}$ and $\mathrm{Ge}$ wafers and reported several deep and shallow levels. However, in both studies, the exact source of the reported defects and their signature were not clarified. In this work, we bridge this knowledge gap by providing the first detailed report on the signature of EDs (in particular, clean $60^{\circ}$ dislocations) in $\mathrm{Ge}_{0.922} \mathrm{Sn}_{0.078}$, epitaxially grown on a Ge substrate using Fourier Transform DLTS. A dislocation is regarded as clean when it exhibits intrinsic properties and is not influenced by external perturbations. These perturbations result from the interaction of a dislocation with point defects, e.g., jogs, kinks or reconstruction defects in the dislocation core, or from impurities segregated in dislocation's long range strain field, in the Cottrell atmospheres. ${ }^{12,17,21}$

Figure 1(a) shows the cross-sectional view of the $p^{-}$ $\mathrm{Ge}_{0.922} \mathrm{Sn}_{0.078} / n^{+} \mathrm{Ge}$ junction diode investigated in this work. $\mathrm{Ge}_{0.922} \mathrm{Sn}_{0.078}$ is grown using chemical vapor deposition $(\mathrm{CVD})^{9}$ on a high quality heavily doped n-type (100) Gewafer $\left(N_{d}^{+}=3.5 \times 10^{18} \mathrm{~cm}^{-3}\right)$. The dopant concentration in the Ge substrate is confirmed using a four-point probe and scanning spreading resistance microscopy (SSRM) measurements. Prior to the epi-growth, the Ge substrate is subjected to an ex-situ $2 \% \mathrm{HF}$ dip and an in-situ bake under $\mathrm{H}_{2}$ at 


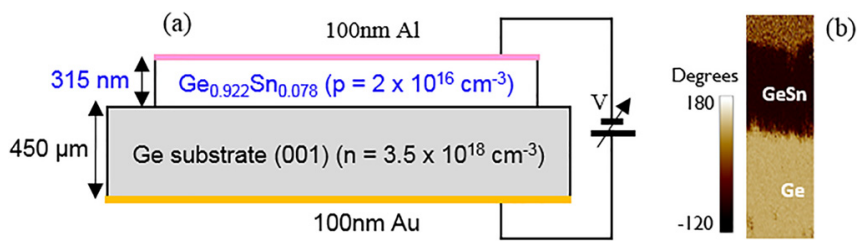

FIG. 1. (a) Cross-sectional schematic. The bias is applied to the Ge substrate. (b) $\delta C / \delta V$ phase values obtained across the cross-section of the sample, using SCM. The scan speed is $0.5 \mathrm{~Hz}$ and the AC bias voltage is $2 \mathrm{~V}$.

$650{ }^{\circ} \mathrm{C}$, to remove any residual oxide. The $\mathrm{Ge}_{0.922} \mathrm{Sn}_{0.078}$ epitaxial layer is subsequently grown at $320^{\circ} \mathrm{C}$ using $\mathrm{Ge}_{2} \mathrm{H}_{6}$ and $\mathrm{SnCl}_{4}$ as precursors and $\mathrm{N}_{2}$ as the carrier gas. The diode is prepared by e-beam evaporation of an $\mathrm{Al}$ circular top contact of $600 \mu \mathrm{m}$ diameter, using a hard-mask. The ohmic contact to the backside of the Ge substrate is made with $100 \mathrm{~nm}$ of $\mathrm{Au}$, deposited by thermal evaporation. The diode area is defined by reactive ion mesa-etching using $\mathrm{SF}_{6}$ and $\mathrm{O}_{2}$ as precursor gases at $100 \mathrm{~W}$ and 80 mTorr, with an etch depth of $\sim 195 \mathrm{~nm}$ and $\mathrm{Al}$ top contacts as the hard mask.

The $\mathrm{Ge}_{0.922} \mathrm{Sn}_{0.078}$ layer is nominally undoped; however, a residual p-type doping is observed. Using scanning capacitance microscopy (SCM), a phase shift of $180^{\circ}$ is obtained between the n-type Ge substrate and the epitaxial layer, indicating that $\mathrm{Ge}_{0.922} \mathrm{Sn}_{0.078}$ has p-type conductivity [Fig. 1(b)]. From the capacitance-voltage $\left(1 / C^{2}-V\right)$ plot (not shown here) at $1 \mathrm{MHz}$ and room temperature (RT), a hole concentration $\left(p=N_{A}^{-}-N_{D}^{+}\right)$of $2 \times 10^{16} \mathrm{~cm}^{-3}$ is extracted for the $\mathrm{Ge}_{0.922} \mathrm{Sn}_{0.078}$ epitaxial layer. $N_{A}^{-}$and $N_{D}^{+}$are the ionized acceptor and compensating donor concentrations, respectively. In comparison, using SSRM, a hole concentration, $p=(3.5$ $\pm 1) \times 10^{16} \mathrm{~cm}^{-3}$ is obtained. It is to be noted that a p-type Ge calibration sample is used for estimating $p$; yet, good agreement is found with more accurate $\mathrm{C}-\mathrm{V}$ measurements.

Figure 2(a) shows the asymmetric reciprocal space map (RSM) acquired around the Ge (224) reciprocal lattice point. The relative position of the Bragg peaks indicates that the $\mathrm{Ge}_{1-\mathrm{x}} \mathrm{Sn}_{\mathrm{x}}$ epitaxial layer is partially relaxed with respect to the Ge substrate. In-plane $\left(a_{L, \|}\right)$ and out-of-plane $\left(a_{L, \perp}\right)$ lattice parameters of the $\mathrm{Ge}_{1-\mathrm{x}} \mathrm{Sn}_{\mathrm{X}}$ epitaxial layer are calculated, using equations from Ref. 22. The substitutional Sn content in $\mathrm{Ge}_{1-\mathrm{x}} \mathrm{Sn}_{\mathrm{x}}$ is calculated by solving Eqs. (1) and (2) re-iteratively until convergence. $\mathrm{b}$ is the bowing parameter with a value of $0.00435 \mathrm{~nm}$ (Ref. 9) and $\nu$ is the Poisson's ratio given by the linear interpolation between the values of $\alpha-\operatorname{Sn}$ and $\operatorname{Ge}[\nu=0.3 x+0.271(1-x)]$

$$
\begin{gathered}
a_{G e S n}=-\frac{a_{L, \perp}(\nu-1)-2 a_{L, \|}(\nu)}{1+\nu}, \\
a_{G e S n}=a_{G e}(1-x)+x a_{S n}+b x(1-x) .
\end{gathered}
$$

The bulk lattice constant, $a_{G e S n}=0.575 \mathrm{~nm}$, is then obtained using Eq. (2). The degree of strain relaxation, $D S R=\left(a_{L, \|}\right.$ $\left.-a_{G e}\right) /\left(a_{G e S n}-a_{G e}\right)$, the misfit $f=\left(a_{G e S n}-a_{G e}\right) / a_{G e}$, and the compressive strain $\varepsilon_{\|}=\left(a_{L, \|}-a_{G e}\right) / a_{G e}$ associated with the $\mathrm{Ge}_{0.922} \mathrm{Sn}_{0.078}$ layer are calculated to be $+58 \%,+1.2 \%$, and $-0.5 \%$, respectively.

In the diamond-like lattice of $\mathrm{Ge}(\mathrm{Sn})$, when the misfit is less than $2 \%$, strain relaxation occurs predominantly by the formation and glide of stable $60^{\circ}$ dislocations. The presence of $60^{\circ}$ misfit dislocations (MD) at the $\mathrm{Ge}_{1-\mathrm{x}} \mathrm{Sn}_{\mathrm{x}} / \mathrm{Ge}$ heterointerface is confirmed by high-resolution TEM (HR-TEM) [Fig. 2(b)]. Figure 2(c) shows the high-angle annular dark fieldscanning TEM image (HAADF-STEM) of the region in the proximity of the $\mathrm{Ge}_{1-\mathrm{x}} \mathrm{Sn}_{\mathrm{x}} / \mathrm{Ge}$ hetero-interface, where the $60^{\circ}$ dislocations are found to be dissociated into Shockley partials ${ }^{12}$ separated by a stacking-fault, of about $5 \mathrm{~nm}-20 \mathrm{~nm}$ wide.

Figure 2(d) shows a HAADF-STEM image of an observed V-shaped defect, with one missing lattice plane on either side. Such V-shaped defects can be associated with (1) a Lomer-Cottrell (L-C) dislocation with a sessile edge character, a reaction product of 2 dissociated Shockley partials, ${ }^{11,23}$ or with (ii) closely spaced pairs of $60^{\circ}$ dislocations with intersecting glide planes and parallel screw components. ${ }^{24}$ However, since the separation between $60^{\circ}$ dislocation pairs can be as small as $3|\vec{b}|$ on average, ${ }^{24}$ the distinction between a L-C dislocation and a $60^{\circ}$ dislocation pair cannot be made. In the statistics of our TEM observations, the V-shaped defects are observed in much lower concentrations as compared to dissociated Shockley partials. Therefore, we use the Burgers vector $(|\vec{b}|)$ of a $60^{\circ}$ dislocation for estimating the dislocation density, using DLTS later in this work. We estimate a TD density of $\sim 2 \times 10^{7} \mathrm{~cm}^{-2}$ from the plan-view TEM image [Fig. 2(e)].
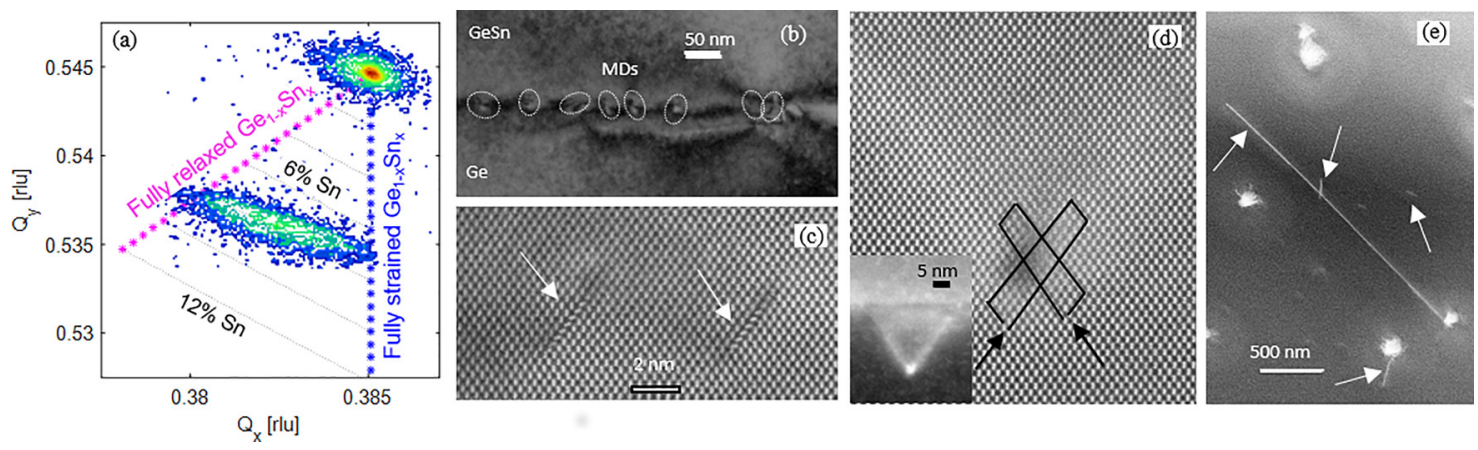

FIG. 2. (a) (224) RSM using an incident beam aligned in the [1 10$]$ direction. $Q_{x}$ and $Q_{z}$ are given in reciprocal lattice units (rlu $=\lambda / 2 d_{h k l}$ ), where $d_{h k l}$ is the spacing between the diffracting planes. Incident $\mathrm{CuK}_{\alpha 1}(\lambda=0.154056 \mathrm{~nm})$ radiation was employed. Loci of points representing the fully relaxed, partially relaxed and fully strained $\mathrm{Ge}_{1-\mathrm{x}} \mathrm{Sn}_{\mathrm{x}}$ layer for various $\mathrm{Sn}$ content are also shown by the (*) symbol. (b) Bright Field [220] cross-sectional TEM showing an array of MDs. (c) HAADF-STEM showing the stacking faults bound by Shockley partials, with an average width of $12 \mathrm{~nm}$. (d) Missing planes on either side of the V-shape defect. [Inset shows the Dark Field (DF)-STEM of the V-shaped defect.] (e) Plan-view DF-STEM showing TDs (marked with arrows). The bright spots are due to surface defects which are not discussed in this work. 
We probe the defect-states in the space charge region (SCR) using DLTS, by applying a quiescent reverse bias $\left(V_{R}\right)$ of $+1 \mathrm{~V}$ and a filling pulse bias $\left(V_{P}\right)$ of $0 \mathrm{~V}$, at the bottom contact to the $n^{+} \mathrm{Ge}$ substrate [see Fig. 1(a)]. At the studied biases, the SCR is varied from $216 \mathrm{~nm}$ to $267 \mathrm{~nm}$, obtained using ( $W=\epsilon_{r} \epsilon_{0} A / C_{R}$ ), where $\mathrm{A}$ is the device area. Due to the low Sn content, the relative permittivity of $\mathrm{Ge}$ $\left(\epsilon_{r}=15.8\right)$ is used for the calculations. ${ }^{25} \epsilon_{0}$ is the vacuum permittivity. The duration of the filling pulse is denoted as $t_{p}$. By using $V_{P} \geq 0$, we ensure that the defect-states capturing majority carriers (holes) in the lowly doped side of a $p^{-} n^{+}$ junction (i.e., $p^{-} \mathrm{Ge}_{0.922} \mathrm{Sn}_{0.078}$ ) are measured.

A hole trap (labeled as H1) is revealed in the DLTS spectra, as shown in Fig. 3(a). Any resolved shoulders or additional peaks are absent, indicating that there is only one dominant type of hole trap, in the explored temperature range.

We attribute the $\mathrm{H} 1$ peak to extended defects, in particular, here $60^{\circ}$ dislocations, based on two observations: (i) asymmetrical peak broadening at the low temperature tail ${ }^{14-16}$ and (ii) dependence of the DLTS signal intensity on $t_{p} .{ }^{16}$ Schröter et al. ${ }^{16,17}$ suggested that defect-states associated with dislocations can be classified into two categories: localized and band-like. The localized states have been associated to the interaction of the dislocation with the point defects, while band-like states are the unperturbed internal states of clean dislocations. ${ }^{16-18}$ The distinction between the two can be made by studying the qualitative features of the DLTS spectra. ${ }^{16,17}$ Evidently, in Fig. 3(a), (i) the peak position shifts to lower temperatures with increasing $t_{p}$ and (ii) the DLTS signals for different $t_{p}$ coincide at the high temperature side. These features of the $\mathrm{H} 1$ defect are characteristic of defect-states with a band-like distribution in the bandgap with rapid inter-state exchange of carriers, ${ }^{16,17}$ as further explained below. Hence, we conclude that the corresponding dislocations are clean. ${ }^{16,17}$

The filling of the defect-states is determined by their position relative to the Fermi-level, $E_{F}$. Under a given bias, $E_{F}$ in $p^{-} \mathrm{Ge}_{0.922} \mathrm{Sn}_{0.078}$ moves closer to the valence bandedge as the temperature decreases [Eq. (3)]. In Eq. (3), $k$ is the Boltzmann constant, $N_{V}$ is the valence band density of states and $E_{V}$ is the position of the valence band edge
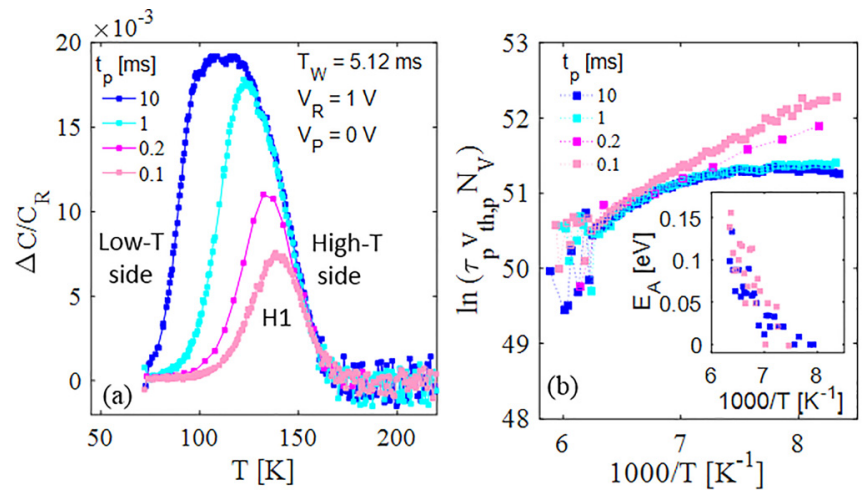

FIG. 3. DLTS spectra of (a) different $t_{p}$, revealing H1 defect. $\Delta C$ is the transient amplitude. $C_{R}$ is the reverse bias capacitance and $T_{W}$ is the window time during which the capacitance transient is recorded. (b) Arrhenius plot for various $t_{p} . \tau_{p}$ is the hole emission time constant. The inset shows the activation energies as extracted from the 1 st order derivative, $\delta\left(\ln \left(\tau_{p} v_{t h . p} N_{V}\right)\right) / \delta(1 / k T)$.

$$
E_{F}=E_{V}+k \operatorname{Tln}\left(N_{V} /\left(N_{A}^{-}-N_{D}^{+}\right)\right) .
$$

As a result, the defect-states lying closer to the valence band edge are filled with holes only at low temperatures. For short $t_{p}$, the low hole-energy states are preferentially filled ${ }^{14,15}$ and at longer $t_{p}$, a higher population of defect-states, including the high hole-energy states are filled. Consequently, the DLTS peak will broaden on its low temperature side and exhibit a higher peak amplitude, as the $t_{p}$ is increased. This is indeed observed [Fig. 3(a)]. Unequivocally, the dominant contribution to the high-temperature edge of the spectra is linked to the low hole-energy states, which are filled for both short and long $t_{p}$. Therefore, the DLTS signal coincides at the high temperature side of the spectra, as observed in Fig. 3(a).

Figure 3(b) shows the Arrhenius plot. For a point defect, one obtains a straight line in an Arrhenius plot and its slope gives the activation energy. However, owing to the extended nature of the $\mathrm{H} 1$ defect, a straight line is not observed in the Arrhenius plot. The activation energies are therefore extracted from the first order derivative, $\delta\left(\ln \left(\tau_{p} v_{t h, p} N_{V}\right)\right) /$ $\delta(1 / k T)$. The highest (electron) energy state $\left(E_{T}\right)$ is found to lie at $\sim E_{V}+0.15 \mathrm{eV}$ and the distribution of defect-states is illustrated in Fig. 4(a). The band diagram is obtained at $V_{R}=0.1 \mathrm{~V}$, at $300 \mathrm{~K}$ using a Sentaurus Device from Synopsys (version J-2014.09). The Sentaurus device is a semi-classical simulator which allows to simulate the device properties, e.g., electrostatics, electrical and optical behavior, using predefined models. For obtaining the band-diagram, it is sufficient to use basic mobility models and the definition of bandgap. Since there is no measurable shift in the position of the valence band at the $\mathrm{Ge} / \mathrm{GeSn}$ interface, ${ }^{26}$ an electron affinity $\left(\chi=E_{g}-E_{V}\right)$ of $4.1 \mathrm{eV}$ is used for the $\mathrm{Ge}_{0.922} \mathrm{Sn}_{0.078}$ layer (at $\epsilon_{\|}=-0.5 \%$ ). The defect-states in Ge are not studied in this work, due to higher n-type doping and therefore are only shown for the illustration purpose in Fig. 4(a).

The association of the $\mathrm{H} 1$ peak with EDs (stacking faults and partials) is further confirmed using isothermal DLTS, where a logarithmic capture behavior is observed over 2 decades (for $t_{p}$ of $1 \mu \mathrm{s}-100 \mu \mathrm{s}$ ) as shown in Fig. 4 (b) $\left(p_{t} / N_{T}\right.$ vs. $t_{p}$ plot). For long $t_{p}$, the holes that are going to be captured experience a repulsive charge due to already captured holes. This built-up charge manifests itself as a capture barrier, $\phi\left(t_{p}\right)$, which increases to a saturation value, when all the defectstates are filled by holes. The $\phi\left(t_{p}\right)$ is extracted using Eq. (4) ${ }^{14}$ and a saturation value of $\sim 60 \mathrm{meV}$ is obtained [Fig. 4(b)]

$$
\phi\left(t_{p}\right)=k T \ln \left(\frac{\left(N_{T}-p_{t}\right) p\left\langle v_{t h, p}\right\rangle \sigma_{p}\left(t_{p}\right)}{d p_{t} / d t_{p}}\right) .
$$

In Eq. (4), $p_{t}$ is the concentration of defect-states occupied by holes, $N_{T}$ is the total defect concentration, $v_{t h, p}$ is the thermal velocity and $\sigma_{p}$ is the hole capture-cross section. $\sigma_{p}$ of $(1.5 \pm 0.2) \times 10^{-19} \mathrm{~cm}^{2}$ is obtained by adopting the procedure described by Omling et al., ${ }^{15}$ from the slope of the linear capture at short $t_{p}$ [Fig. 4(c)]. A hole mass of $0.22 m_{0}$ (at $\left.\epsilon_{\|}=-0.5 \%\right)^{27}$ is used to calculate $v_{t h, p}$, where $m_{0}$ is the electron rest mass. Since the temperature dependence of the hole mass of $\mathrm{Ge}_{1-\mathrm{x}} \mathrm{Sn}_{\mathrm{x}}$ is not known, the hole mass at RT is used for the calculation. 
(a)

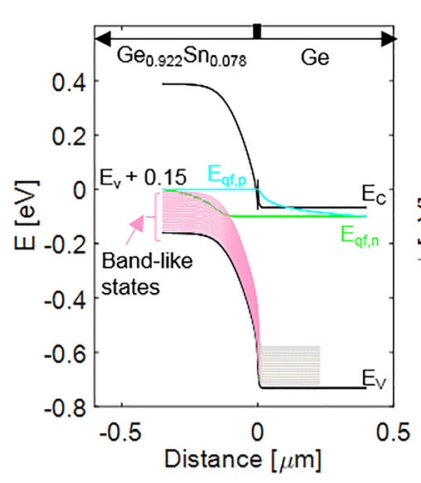

(b)

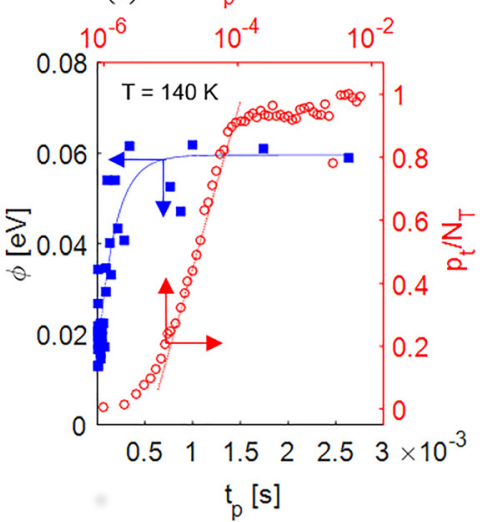

(c)

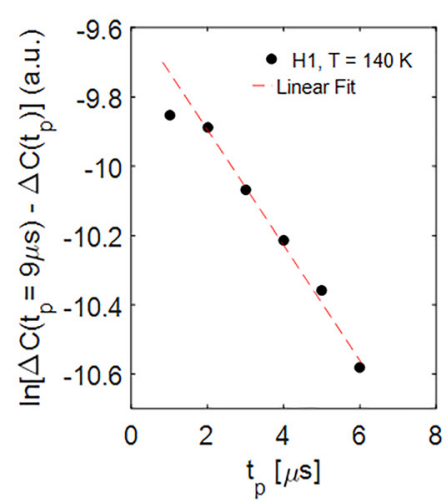

(d)

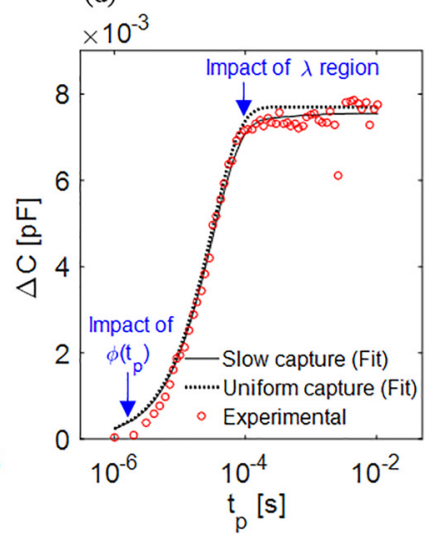

FIG. 4. (a) Schematic of the spread of band-like states associated with $\mathrm{H} 1$ defect in the bandgap of the $\mathrm{Ge}_{0.922} \mathrm{Sn}_{0.078}$ epitaxial layer. (b) Capture kinetics at constant temperature $\left(T=140 \mathrm{~K}, \Delta T<0.1 \mathrm{~K}, V_{R}=1 \mathrm{~V}, V_{P}=0 \mathrm{~V}, T_{W}=5.12 \mathrm{~ms}\right)$. Occupied defect-states, $p_{t}$, normalized with respect to the total concentration, $N_{T}$, as a function of the filling pulse duration $t_{p}$ (red circles) and Coulomb barrier height $(\phi)$ evaluated vs. $t_{p}$ (blue squares). The dotted line is only a guide for the eye. (c) Hole capture behavior for short $t_{p}$ for the H1 defect. (d) Simulated capture kinetic of a point defect with slow capture and with uniform capture (without slow capture). Experimental data (empty circles) for H1 defect are shown for comparison.

Logarithmic capture behavior can also be observed for point defects in the case of slow capture in the Debye-tail of carriers (the $\lambda$-region). ${ }^{13,28}$ This effect, however, cannot explain the logarithmic $t_{p}$ dependence observed in Fig. 4(b) in the $1 \times 10^{-6} \mathrm{~s}$ to $1 \times 10^{-4} \mathrm{~s}$ range, as we show through simulations. The simulation algorithm includes the effect of both slow capture and carrier re-emission. ${ }^{28}$ Figure 4(d) shows two simulated curves for capture by point defects: one corresponding to the uniform trapping rate and one including slow capture in the $\lambda$ region. It is clear that the impact of slow capture is mainly observable for $t_{p}$ values greater than $1 \times 10^{-4} \mathrm{~s}$. Furthermore, for shorter $t_{p}\left(<1 \times 10^{-5} \mathrm{~s}\right)$, both models for point defects disagree with experiment, highlighting the effect of the capture barrier $\phi\left(t_{p}\right)$. H1 should therefore indeed be interpreted as an extended defect.

The small capture cross-section suggests that the H1 defect acts as a donor-like repulsive center. Due to the donor-like nature, H1 defect-states would act as compensating donors $\left(N_{D}^{+}\right)$. Therefore, the nature and the properties of shallow defects leading to p-type unintentional doping $\left(p=N_{A}^{-}-N_{D}^{+}\right)$remain unknown. However, positron annihilation spectroscopy studies associate p-type doping with vacancy clusters in epitaxial $\mathrm{Ge}_{\mathrm{x}} \mathrm{Sn}_{1-\mathrm{x}}{ }^{29}$ These vacancies can be introduced owing to non-equilibrium epitaxial growth $^{20}$ and the movement of dislocations via jogs. ${ }^{12}$

The concentration of the H1 defect can be estimated using the peak amplitude of the DLTS spectra [Fig. 3(a)], corresponding to the longest filling pulse $\left(t_{p}=10 \mathrm{~ms}\right)$, which is the representative of complete occupation of defect-states. The complete occupation of defect-states (i.e., $p_{t}=N_{T}$ ) at $t_{p}=10 \mathrm{~ms}$ is indicated by the plateau in the capture kinetics, as shown in Fig. 4(b). A $N_{T}$ value of $1.1 \times 10^{15} \mathrm{~cm}^{-3}$ is obtained using Eq. (5). Here, the pulse correction factor, PCF $\approx\left(V_{R}+V_{b i}\right)$ / $\left(V_{R}-V_{P}\right)$, employs the $\lambda$-correction for the capture at the edge of the depletion region. ${ }^{13,30} V_{b i}$ is the built-in potential

$$
N_{T}=2\left(N_{A}^{-}-N_{D}^{+}\right) \frac{\Delta C}{C_{R}} P C F .
$$

A dislocation density $\left(\rho_{T}\right)$ of $\sim 4.2 \times 10^{7} \mathrm{~cm}^{-2}$ is obtained under the assumption of one state per inter-atomic distance along a $60^{\circ}$ dislocation $\left(\rho_{T}=N_{T} \cdot|\vec{b}|\right)$. The magnitude of $\rho_{T}$ is comparable to that measured using a TEM $\left(2 \times 10^{7} \mathrm{~cm}^{-2}\right)$.

The direct- $\Gamma$ and indirect- $\mathrm{L}$ bandgaps of the $\mathrm{Ge}_{0.922} \mathrm{Sn}_{0.078}$ layer are calculated to be equal to $0.585 \mathrm{eV}$ and $0.549 \mathrm{eV}$, respectively. ${ }^{6}$ Although $\mathrm{Ge}_{0.922} \mathrm{Sn}_{0.078}$ at the given strain and Sn content is an indirect semiconductor, a significant increase in the reverse-bias current density $\left(J_{R}\right)$ associated with minority electrons is observed above $160 \mathrm{~K}$ [Fig. 5(a)]. From the Arrhenius analysis of the reverse bias current, an activation energy $\left(E_{A}\right)$ of $0.42 \mathrm{eV}$ is obtained [Fig. $\left.5(\mathrm{~b})\right]$ for $T>160 \mathrm{~K}$. We suggest that the $E_{A}$ of $0.42 \mathrm{eV}$ is associated with the SRH generation of minority electrons via the band of defect-states, since $E_{C}-E_{T} \simeq 0.43 \mathrm{eV}$, as shown in the inset of Fig. 5(b). Below $125 \mathrm{~K}$, as shown in Fig. 5(b), the current is nearly independent of temperature and is possibly associated with conduction of carriers in the dislocation-states followed by defect-state to band tunneling. ${ }^{10}$ In the transition temperature range, $125-160 \mathrm{~K}$, the slope continuously changes, and therefore an activation energy in the range of $0.16 \mathrm{eV}-0.26 \mathrm{eV}$ is obtained. However, peaks in addition to $\mathrm{H} 1$ are not observed in the DLTS spectra [Fig. 3(a)], suggesting that the

$\mathrm{T}[\mathrm{K}]$
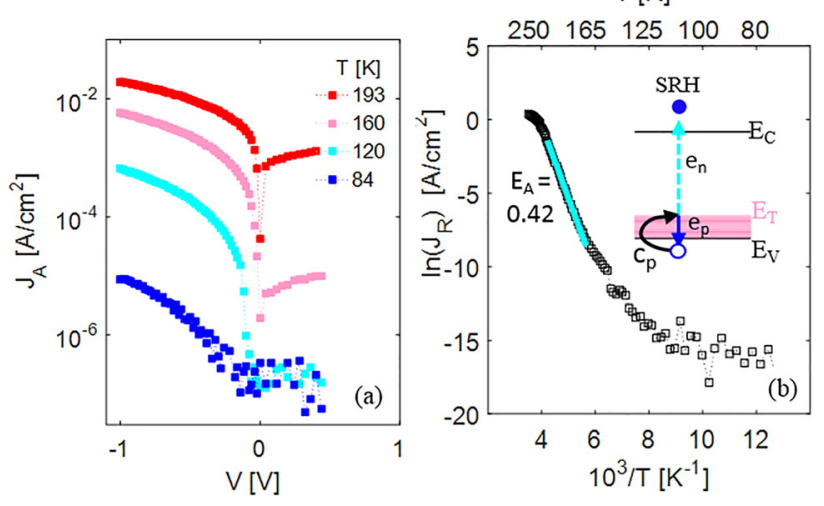

FIG. 5. (a) Current-voltage (I-V) characteristics normalized with respect to area $\left(J_{A}\right)$ at different temperatures. (b) Arrhenius plot of the reverse-bias current density $\left(J_{R}\right)$ at $V=0.4 \mathrm{~V}$. The inset shows a schematic of minority carrier generation via the SRH mechanism from the band-like states of $\mathrm{H} 1$ defect in the GeSn bandgap. $e_{n}$ is the electron emission rate, and $c_{p}$ and $e_{p}$ are the hole capture and emission rates, respectively. 
intermediate activation energy is associated with a minority carrier (electron) defect in $p^{-} \mathrm{Ge}_{0.922} \mathrm{Sn}_{0.078}$. Further investigations will be needed using injection-DLTS.

In conclusion, clean $60^{\circ}$ dislocations lead to donor-like repulsive states with a band-like distribution in the bandgap of $\mathrm{Ge}_{0.922} \mathrm{Sn}_{0.078}$. These $60^{\circ}$ dislocations are found to be split into Shockley partials, bound by a stacking fault. They are not responsible for the unintentional p-type doping in $\mathrm{Ge}_{0.922} \mathrm{Sn}_{0.078}$. However, they are electrically active and generate excess current in the junction diode, via the SRH mechanism. We speculate that the nature of the $60^{\circ}$ dislocations will not change considerably with the Sn content and the DSR; therefore, it is imperative to prevent their propagation to the active regions.

${ }^{1}$ J. Kouvetakis, J. Menendez, and A. Chizmeshya, Annu. Rev. Mater. Res. 36, 497 (2006).

${ }^{2}$ S. Zaima, O. Nakatsuka, N. Taoka, M. Kurosawa, W. Takeuchi, and M. Sakashita, Sci. Technol. Adv. Mater. 16, 043502 (2015).

${ }^{3}$ R. Loo, B. Vincent, F. Gencarelli, C. Merckling, A. Kumar, G. Eneman, L. Witters, W. Vandervorst, M. Caymax, M. Heyns, and A. Thean, ECS J. Solid State Sci. Technol. 2, N35 (2013).

${ }^{4}$ G. Eneman, D. Brunco, L. Witters, B. Vincent, P. Favia, A. Hikavyy, A. De Keersgieter, J. Mitard, R. Loo, A. Veloso, O. Richard, H. Bender, S. Lee, M. Van Dal, N. Kabir, W. Vandervorst, M. Caymax, N. Horiguchi, $\mathrm{N}$. Collaert, and A. Thean, in IEEE International Electron Devices Meeting (IEDM) (IEEE, 2012), pp. 5-6.

${ }^{5}$ S. Wirths, R. Geiger, N. Von Den Driesch, G. Mussler, T. Stoica, S. Mantl, Z. Ikonic, M. Luysberg, S. Chiussi, J. Hartmann, H. Sigg, J. Faist, D. Buca, and D. Grützmacher, Nat. Photonics 9, 88 (2015).

${ }^{6}$ Y. Shimura, S. A. Srinivasan, and R. Loo, ECS J. Solid State Sci. Technol. 5, Q140 (2016).

${ }^{7}$ C. Schulte-Braucks, R. Pandey, R. N. Sajjad, M. Barth, R. K. Ghosh, B. Grisafe, P. Sharma, N. von den Driesch, A. Vohra, G. B. Rayner, R. Loo, S. Mantl, D. Buca, C.-C. Yeh, C.-H. Wu, W. Tsai, D. A. Antoniadis, and S. Datta, IEEE Trans. Electron Devices 64, 4354 (2017).

${ }^{8}$ S. Assali, A. Dijkstra, A. Li, S. Koelling, M. Verheijen, L. Gagliano, N. von den Driesch, D. Buca, P. Koenraad, J. Haverkort, and E. P. A. M. Bakkers, Nano Lett. 17, 1538 (2017).
${ }^{9}$ F. Gencarelli, "Epitaxial growth of GeSn compounds for advanced CMOS and photonics applications," Ph.D. thesis, KU Leuven, 2015.

${ }^{10}$ M. Reiche, M. Kittler, H. Übensee, M. Krause, and E. Pippel, Jpn. J. Appl. Phys., Part 1 53, 04EC03 (2014).

${ }^{11}$ P. Petroff, R. Logan, and A. Savage, J. Microsc. 118, 255 (1980).

${ }^{12}$ D. B. Holt and B. G. Yacobi, Extended Defects in Semiconductors: Electronic Properties, Device Effects and Structures (Cambridge University Press, 2007).

${ }^{13}$ P. Blood and J. W. Orton, The Electrical Characterization of Semiconductors: Majority Carriers and Electron States (Academic Press, 1992), Vol. 2.

${ }^{14}$ P. Grillot, S. Ringel, E. Fitzgerald, G. Watson, and Y. Xie, J. Appl. Phys. 77, 3248 (1995).

${ }^{15}$ P. Omling, E. Weber, L. Montelius, H. Alexander, and J. Michel, Phys. Rev. B 32, 6571 (1985).

${ }^{16}$ W. Schröter, J. Kronewitz, U. Gnauert, F. Riedel, and M. Seibt, Phys. Rev. B 52, 13726 (1995).

${ }^{17}$ W. Schröter, H. Hedemann, V. Kveder, and F. Riedel, J. Phys.: Condens. Matter 14, 13047 (2002).

${ }^{18}$ O. Vyvenko, M. Kittler, W. Seifert, and M. Trushin, Phys. Status Solidi (c) 2, 1852 (2005).

${ }^{19}$ M.-Y. Ryu, Y. Yeo, M. Ahoujja, T. Harris, R. Beeler, and J. Kouvetakis, Appl. Phys. Lett. 101, 131110 (2012).

${ }^{20}$ W. Takeuchi, T. Asano, Y. Inuzuka, M. Sakashita, O. Nakatsuka, and S. Zaima, ECS J. Solid State Sci. Technol. 5, P3082 (2016).

${ }^{21}$ P. Hirsch, J. Microsc. 118, 3 (1980).

${ }^{22}$ T. Roesener, V. Klinger, C. Weuffen, D. Lackner, and F. Dimroth, J. Cryst. Growth 368, 21 (2013).

${ }^{23}$ Y. B. Bolkhovityanov, A. Deryabin, A. Gutakovskii, and L. Sokolov, Acta Mater. 61, 617 (2013).

${ }^{24}$ J. Narayan and S. Oktyabrsky, J. Appl. Phys. 92, 7122 (2002).

${ }^{25}$ M. Oehme, K. Kostecki, K. Ye, S. Bechler, K. Ulbricht, M. Schmid, M. Kaschel, M. Gollhofer, R. Körner, W. Zhang et al., Opt. Express 22, 839 (2014).

${ }^{26}$ V. Afanas'ev, C. Schulte-Braucks, S. Wirths, J. Schubert, and D. Buca, Microelectron. Eng. 178, 141 (2017).

${ }^{27}$ C. Schulte-Braucks, D. Stange, N. Von Den Driesch, S. Blaeser, Z. Ikonic, J. Hartmann, S. Mantl, and D. Buca, Appl. Phys. Lett. 107, 042101 (2015).

${ }^{28}$ J. Lauwaert, J. Van Gheluwe, and P. Clauws, Rev. Sci. Instrum. 79, 093902 (2008).

${ }^{29}$ J. Slotte, F. Tuomisto, J. Kujala, A. M. Holm, N. Segercrantz, S. Kilpeläinen, K. Kuitunen, E. Simoen, F. Gencarelli, R. Loo, and Y. Shimura, ECS Trans. 64, 241 (2014).

${ }^{30}$ E. Simoen, P. Clauws, and J. Vennik, J. Phys. D: Appl. Phys. 18, 2041 (1985). 